\title{
Spectroscopy of the symbiotic binary CH Cygni from 1996 to 2007
}

\author{
M. Burmeister ${ }^{1,2}$ and L. Leedjärv ${ }^{1}$ \\ 1 Tartu Observatory, 61602 Tõravere, Estonia \\ e-mail: mari@aai .ee \\ 2 Institute of Physics, University of Tartu, Tähe 4, 51010 Tartu, Estonia
}

Received 20 January 2009 / Accepted 2 June 2009

\begin{abstract}
Context. Efforts to uncover the nature of the variable star CH Cyg have lasted for decades by now. Recently, the model of a red giant and an accreting white dwarf on a $~ 5700$-day orbit has seemed to have gained an advantage over other ideas, mainly as a result of the investigation of the cool component's absorption lines. In addition to those lines the star's spectrum also presents emission features, which form near the hot component and are therefore a probe of the accretion phenomenon, which in turn could be bound to the orbital motion.

Aims. We have monitored a set of emission lines in the optical spectra of $\mathrm{CH}$ Cyg over an extended period of time to determine relations between the behaviour of those lines and the photometric data. We are searching for a possible connection with the star's orbital motion, and find hints of the physical phenomena occurring near the white dwarf.

Methods. More than 500 observations of CH Cyg were carried out during 1996-2007 using the 1.5-m telescope at the Tartu Observatory, Estonia. Most of the spectra were registered in the $\mathrm{H} \alpha$ region, but other Balmer lines and lines of $\mathrm{He}, \mathrm{N}, \mathrm{O}$, and $\mathrm{Fe}$ were also investigated in terms of their equivalent widths, radial velocities, and absolute fluxes.

Results. The spectra indicate different stages that $\mathrm{CH}$ Cyg has been through in the course of our observations. During quiescence, the strength of the line profiles changes in opposite phase with the star's luminosity. The $\mathrm{H} \alpha$ profile becomes double-peaked at times, which may point to the temporary presence of some disk-like structure, but also to absorption in neutral gas surrounding the area of the formation of the emission component. Occasionally the line develops wide wings, which indicate high velocities of the matter. In 1999, when a periastron passage is assumed according to the 5700-day model, the Balmer lines strengthen considerably, as might be expected due to an increase in the accretion rate. The eclipse in 2001, however, is not noticed. At certain episodes, the controversial 756-day period of $\mathrm{CH}$ Cyg is seen in our data.
\end{abstract}

Key words. binaries: symbiotic - stars: individual: CH Cyg

\section{Introduction}

$\mathrm{CH}$ Cygni is a variable star that has been studied extensively for a long time and over a wide spectral range (see Mikołajewski et al. 1990a for a historical review). The star has for some time been included in the group of symbiotic stars and is one of the brightest among them with its average visual magnitude of about 7.1 (Belczyński et al. 2000). It is also one of the nearest symbiotics with a distance of $268 \pm 62$ pc measured by Hipparcos (Munari et al. 1997).

For a long time, however, $\mathrm{CH}$ Cyg was considered a single red giant experiencing low-amplitude pulsations with a period around 100 days. It did not show any spectral peculiarities and was even adopted as a standard star of the spectral class M6 III in the Morgan-Keenan classification, and yet in 1963 a strong blue continuum and HI emission lines appeared in the star's spectrum (Deutsch 1964). At that time, the spectrum of CH Cyg resembled a symbiotic star. This phenomenon was observed again in 1965. Since then, there have been several such outbursts of different durations: 1967-1970, 1977-1986, 1992-1995, and 1998-1999, separated by quiescent periods, during which the spectrum of $\mathrm{CH}$ Cyg resembled that of a single red giant, like before 1963. According to its infrared colours, CH Cyg is classified as an S-type symbiotic. However, its orbital period appears to be unusually long for that class, and sometimes it shows strong [N II] emission lines that are more characteristic of the D-type systems.
During the longest and most pronounced outburst in 1977-1986, a sudden drop in visual light was recorded in 1984, followed by the formation of a jet detected at radio wavelengths (Taylor et al. 1986). In 1996, after a minor outburst, the deepest minimum of visual light $\left(V \sim 10^{\mathrm{m}}\right)$ ever recorded took place (e.g., Mikołajewski et al. 1996). A radio map obtained by Karovska et al. (1998) in January 1997 revealed an elongated structure, which in their opinion could be related to jet formation. Crocker et al. (2001) found jet-like extensions in their radio images obtained in 1999 during the 1998-1999 outburst.

$\mathrm{CH}$ Cyg is one of the few symbiotics demonstrating flickering on a timescale of minutes (Dobrzycka et al. 1996). Simultaneously with the appearance of the jets, the disappearance of the flickering and the enhancement of the blue continuum has been observed, as well as sudden broadening of emission lines.

About ten symbiotic systems have demonstrated jets by now (Brocksopp et al. 2004; Leedjärv 2004). The mechanism of jet formation is not clear, but it is suspected that it is related to an accretion disk around the compact component. In this case, some symbiotics may have at least transient accretion disks. The disappearance of flickering after jet formation could then point to the disruption of the disk (Sokoloski \& Kenyon 2003).

It is generally believed that the activity of $\mathrm{CH}$ Cyg is powered by the energy generated by the accretion of the red giant wind by the hot component as the luminosity of the latter (a few 
hundred $L_{\odot}$ at the highest) seems to be too low to be caused by a nuclear-burning shell. The amount of the accreted matter may depend on the pulsations of the giant, as well as on the orbital motion of the stars, if the orbit is elliptical.

When it comes to precise details of the system, no generally accepted view exists, although several possible models have been presented. A magnetic rotator model was proposed by Mikołajewski et al. (1990b). According to them, CH Cyg contains a pulsating red giant and a white dwarf with a strong magnetic field on an eccentric long-period orbit. Mikołajewski et al. (1990a) propose an orbital period of 5700 days.

Hinkle et al. (1993) discovered very regular variations with a 756-day period from the radial velocities in the infrared spectra of $\mathrm{CH} \mathrm{Cyg}$, which they interpreted as the orbital period of the symbiotic binary. Their model also involved a $\mathrm{G}$ dwarf as a third component, orbiting the binary with a 5300-day period. This model found both improvement (Skopal 1995; Skopal et al. 1996b) and opposition (Munari et al. 1996). The last authors suggested that the 756-day modulation is caused by the pulsation of the giant, rather than by orbital motion. In light of new observations and the improved estimates of system parameters, Hinkle et al. (2009) renounce their triple-star model and suggest an orbital period of 5689 days for the symbiotic pair, close to the periods in former binary models.

We present our spectroscopic observations of $\mathrm{CH}$ Cyg at Tartu Observatory from 1996 to 2007. In Sect. 2 we describe our observational equipment, the data, reduction tools, and measurements. The overview of the star's photometric and spectroscopic variations follows in Sects 3 and 4, respectively. Analysis of the results is presented in Sect. 5 .

\section{Observations and data reduction}

All the spectra treated in this paper were obtained at Tartu Observatory using the $1.5 \mathrm{~m}$ telescope equipped with the Cassegrain grating spectrograph. Until 1999 March, the detector was the SpectraSource Instruments CCD camera HPC-1 (Tek $1024 \times 1024$ chip, pixel size $24 \times 24 \mu \mathrm{m}$, Peltier cooled). From 1999 March to 2006 March, the spectra were recorded with the cryogenically (liquid nitrogen) cooled CCD camera Orbis-1 of the same company (Tek $512 \times 512$ chip, pixel size $24 \times 24 \mu \mathrm{m}$ ). Since then we have used the Andor Technologies CCD camera Newton DU-970N $(400 \times 1600$ chip, pixel size $16 \times 16 \mu \mathrm{m}$, Peltier cooled).

From 1996 to 2007, almost 500 spectra were obtained, which cover the time interval without major gaps. In general, the spectra can be divided into two categories: red and blue. Most of the red spectra were made with an 1800 lines-per-mm (lpm) grating and linear dispersion of either $0.29 \AA \mathrm{px}^{-1}$ (HPC- 1 and Orbis-1 cameras), or $0.19 \AA \mathrm{px}^{-1}$ (Newton camera). An 1200 lpm grating was used for the rest of the spectra in the red region, which therefore have lower resolution. All the spectra in the blue region were made with the $1200 \mathrm{lpm}$ grating. Table 1 lists the wavelength coverages with corresponding lines for each camera. The high-resolution red spectra contain the $\mathrm{H} \alpha$, [N II] 6548 , and 6584 lines. Velocities of these lines could be measured with an accuracy of $3-4 \mathrm{~km} \mathrm{~s}^{-1}$ (HPC-1 and Orbis-1) or $2.5-3 \mathrm{~km} \mathrm{~s}^{-1}$ (Newton). The accuracy was lower for the rest of the lines, $\sim 15 \mathrm{~km} \mathrm{~s}^{-1}$ at worst. The [O I] 6300 and 6364 lines are found in the red spectra with lower resolution. The blue spectra made with Orbis- 1 camera include either $\mathrm{H} \beta$ with [O III] 4959 and 5007 lines or $\mathrm{H} \gamma$, [O III] 4363, and $\mathrm{H} \delta$ lines. Blue spectra by Newton DU-970N contain all these lines, except $\mathrm{H} \delta$. Certain
Table 1. Wavelength regions, covered by our spectra with different cameras.

\begin{tabular}{lll}
\hline \hline HPC-1 & Orbis-1 & Newton DU-970N \\
\hline $\mathrm{H} \alpha(1800)$ & $\mathrm{H} \alpha(1800)$ & $\mathrm{H} \alpha(1800)$ \\
$6500-6750 \AA$ & $6490-6620 \AA$ & $6475-6740 \AA$ \\
$\mathrm{H} \alpha(1200)$ & $\mathrm{H} \alpha(1200)$ & $\mathrm{H} \alpha(1200)$ \\
$6200-6900 \AA$ & $6230-6620 \AA$ & $6130-6900 \AA$ \\
$\mathrm{H} \beta$ & $\mathrm{H} \beta$ & $\mathrm{H} \gamma-\mathrm{H} \beta$ \\
$4200-5050 \AA$ & $4610-5040 \AA$ & $4200-5120 \AA$ \\
& $\mathrm{H} \delta-\mathrm{H} \gamma$ & \\
& $3930-4400 \AA$ \\
\hline
\end{tabular}

lines of neutral helium $(\lambda \lambda 4026,4388,4471$, and 6678) fall into some of our spectra as well.

The spectra were reduced using the software package MIDAS provided by ESO, except some earlier spectra, for which a software package KASPEK, developed at Tartu Observatory, was applied. After subtracting the dark frame (in the case of HPC-1 spectra) or bias (Orbis-1 spectra) and sky background, the spectra were calibrated to the wavelength scale using an $\mathrm{Ne}$ Ar (until 1999 October) or Th-Ar (since 1999 October) hollow cathode lamp. The calibrated spectra were normalised to the continuum. The following properties of emission lines were measured: peak intensity, equivalent width, velocity, and full width at half maximum, of which the first three are treated in this paper.

Remark. In the figures of this paper we also present some data that have been obtained before the period under discussion, 1996-2007. Their purpose is illustrative; no high-resolution spectra suitable for more precise investigation can be found among them.

\section{Photometric variability of $\mathrm{CH}$ Cygni}

As we did not perform any photometry ourselves, the photometric data necessary for calculating the absolute fluxes of lines (Sect. 4.6) were gathered from the literature (Skopal et al. 1995, 1996a, 2000, 2002, 2004, 2007; Skopal 1997, 1998; Hric et al. 1996; Leedjärv \& Mikołajewski 2000). The $U B V$ values are presented in Fig. 1. We do not possess any observations after January 2007, but the visual estimates by AAVSO show that the $V$ brightness of the star that faded to 10th magnitude by the end of 2006 and remained on that low level throughout 2007. According to Taranova \& Shenavrin (2006), the fading was particularly remarkable in the infrared spectral region, as by the end of 2006 the $J$ magnitude of $\mathrm{CH}$ Cyg was about 0.7 mag fainter than during the previous, similar event that occurred in 1996. Possibly a new rise started in October 2007.

Photometric observations have shown the luminosity of $\mathrm{CH}$ Cyg to be variable on both short and long timescales. Several periods have been found from the stars' light curves, though none of them is obvious. The shortest of these periods (minutes or hours) are generally associated with the activity of the hot component and the longer ones (starting from 100 days) with the pulsation and rotation of the giant, as well as the orbital motion of the binary. Among the longer periods, those of 756 and 5700 days have gained special interest, especially as similar periods have been found from the red giant absorption features (Hinkle et al. 1993). The shortest one of these resembles the orbital periods of other symbiotic stars, but is not always present 
Year

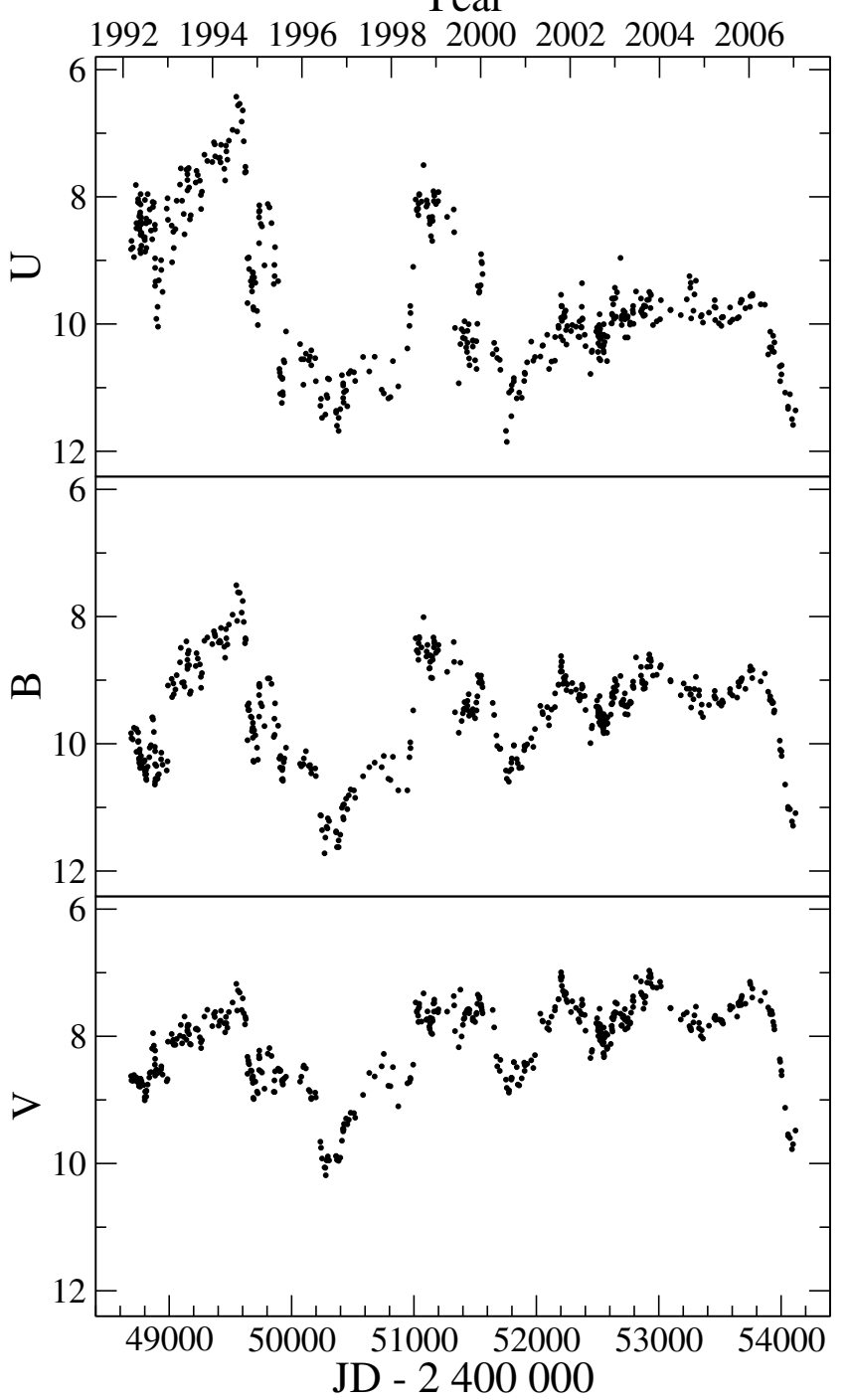

Fig. 1. Recent $U, B$, and $V$ data of $\mathrm{CH} C y g$, all on the same scale for easier comparison.

in the light curve. The period of $\sim 5700$ days, first reported by Yamashita \& Maehara (1979), is possibly related to the orbital motion, although this is exceptionally long for an S-type symbiotic star.

\section{Variations in the spectrum: equivalent widths and radial velocities}

Symbiotic stars are described as having emission lines in their spectra, but the prominence of these lines varies from system to system (see the spectra of different symbiotics in Munari \& Zwitter 2002). The emission line spectrum of $\mathrm{CH}$ Cyg is relatively undistinguished. Most of the time, only the Balmer lines are easily found; the lines of neutral helium are very weak and high excitation lines tend to be missing. In the red part of the optical spectrum, molecular absorption of the $\mathrm{M}$ giant can be seen. However, the emission spectrum becomes more conspicuous at certain episodes. As our observations cover the interval 1996-2007, they include the last outburst in 1998-1999 and the more or less quiescent time since then. Balmer lines were strong during the outburst and the light minima in 1996 and (a)
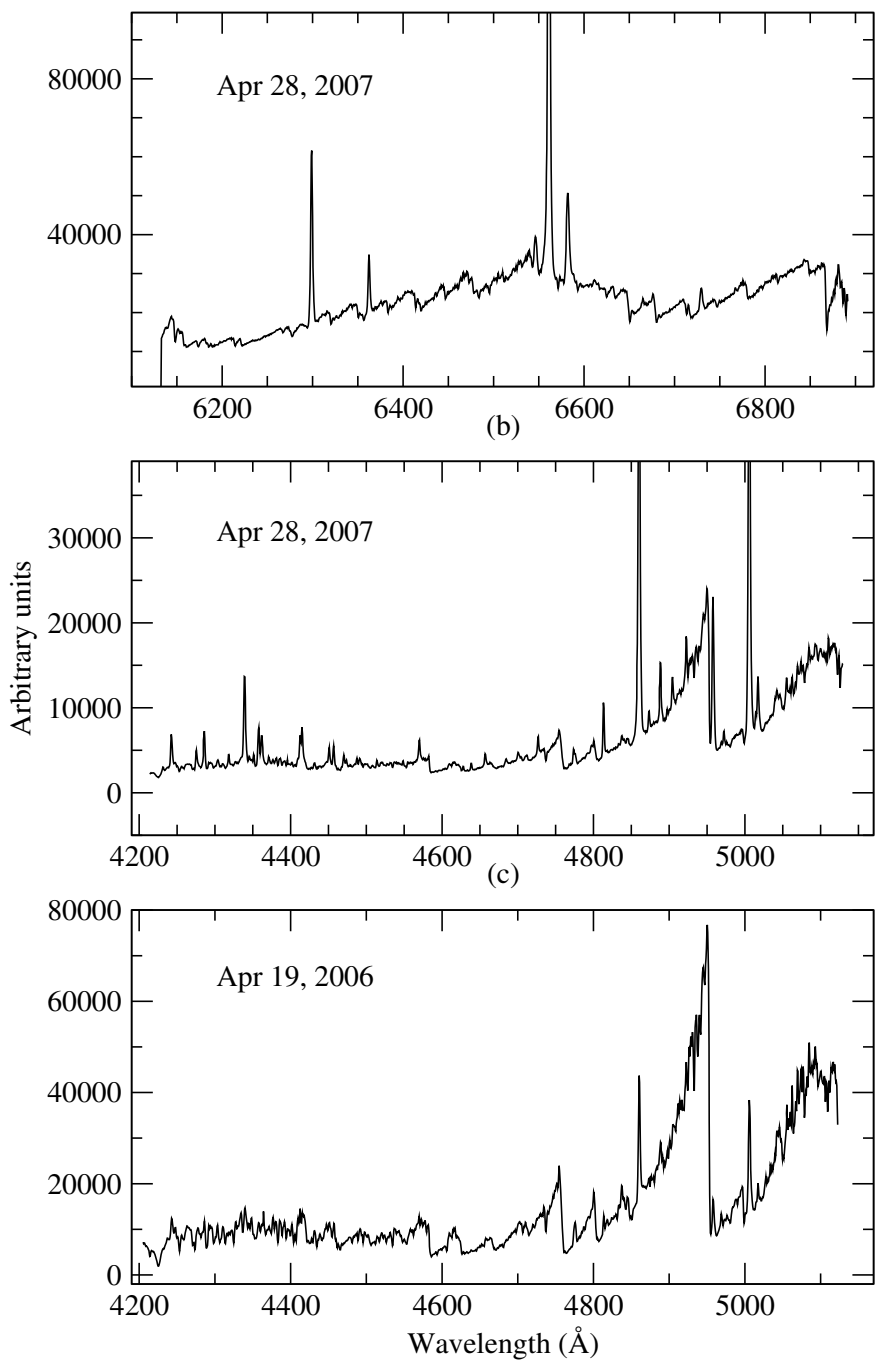

Fig. 2. Representative lower-resolution spectra from 2007 April 28, when the continuum was weak and the emission lines exceptionally conspicuous (a) and b)), and from 2006 April 19 c), when the emission lines were weak. Most of the time of our observations the spectra of $\mathrm{CH}$ Cyg looked like the latter.

2006-2007, with strong forbidden lines seen only during the mentioned minima. Figure 2 shows representative spectra from red and blue regions, made with Newton DU-970N.

\subsection{Balmer lines}

Our main interest was concentrated on the $\mathrm{H} \alpha$ line, the strongest of the emission lines in CH Cyg spectrum. Most of the spectra in the region of that line were made with a resolution high enough to allow the investigation of the fine structure of the line. During the time interval under consideration, the star was mostly quiescent $\left(U \geq 10^{\mathrm{m}}\right)$, however, activity occurred before 1996 and in 1998-2000. The intensity and shape of the $\mathrm{H} \alpha$ emission line were highly variable, and sometimes the line became doublepeaked. Figure 3 shows some examples of the $\mathrm{H} \alpha$ line profile at different times. When the line is double-peaked, the two emission components often have very different heights, the red peak generally being stronger than the blue one. Single-peaked line profiles are usually asymmetric with a step on the blue side, 


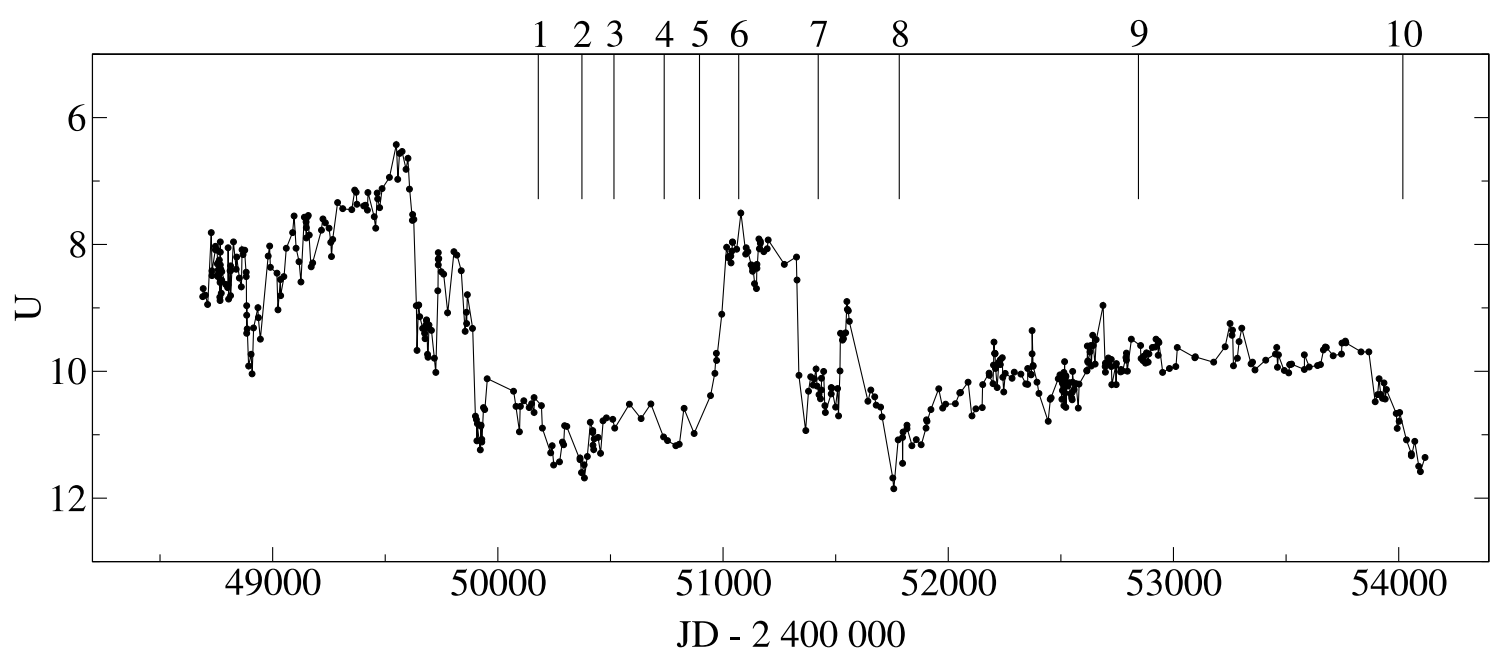

(1)

(2)

(3)

(4)

(5)

(6)

(7)

(8)

(9)

(10)

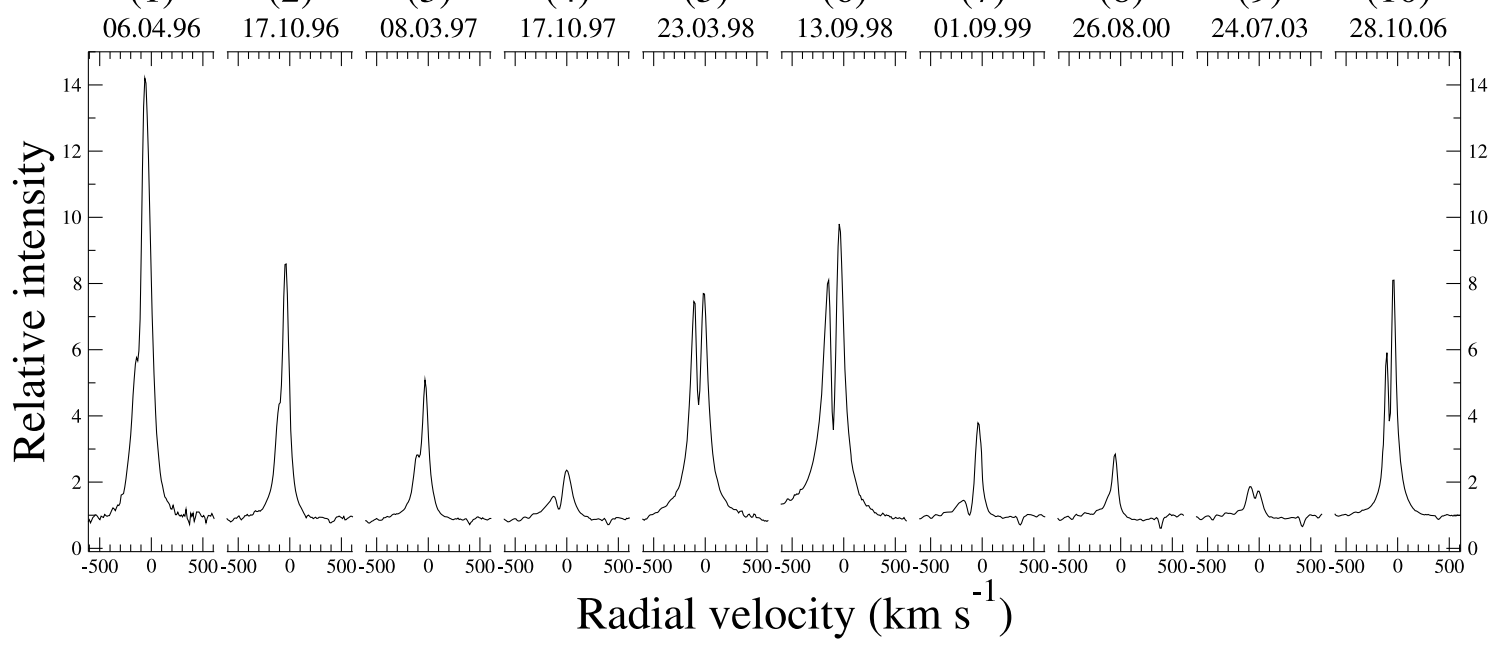

Fig. 3. Top: recent $U$ light curve of CH Cyg. Numbered positions correspond to the spectra below. Bottom: some examples of the H $\alpha$ profile of $\mathrm{CH}$ Cyg, all plotted on the same scale for comparison.

alluding to a blue-shifted absorption component. These shapes can occur in cases of both weak and strong lines.

Below we characterise the $\mathrm{H} \alpha$ line variations between 1996 and 2007, using equivalent width (EW) as a measure of its strength, and relying mostly on the spectra taken with the $1800 \mathrm{lpm}$ grating (see Sect. 2). After the qualitative description of the line profiles' evolution, the quantitative evaluation of the radial velocities is given.

The spectra in early 1996 showed a strong $\mathrm{H} \alpha$ line, which had a single-peaked profile with a slight step on the blue side (see the first line in Fig. 3). During the second half of the year, the line was considerably weaker (second line), but had a similar shape.

In $1997 \mathrm{EW}$ of the line continued to vary, but was on average lower than the year before. The shape of the profile remained almost unchanged, that is, single-peaked and asymmetric. For some time in October when the line was very weak (fourth line in Fig. 3), the step on the blue side deepened into an absorption component.

In early 1998 the $\mathrm{H} \alpha$ line was still weak, but developed wide wings (fifth line in Fig. 3) by March, i.e. before the increase in brightness, which started in the middle of the year. In August and September the line was at its highest intensity (sixth line in Fig. 3) and at the same time asymmetric, with the blue wing extending to velocities of $-1100 \mathrm{~km} \mathrm{~s}^{-1}$. The red wing, in contrast, remained relatively moderate with a velocity of about $400 \mathrm{~km} \mathrm{~s}^{-1}$. The profile had two peaks of comparable strength for most of the year, but the blue peak declined during the last months.

Rapid changes took place in 1999. During the first months, the $\mathrm{H} \alpha$ line was still strong and double-peaked, varying rapidly at the same time (Fig. 4, left panel). Multiple absorption components in the blue wing, seen already in December 1998, became more prominent by May 1999. On May 31, two absorption components were clearly visible, as shown in Fig. 4 (right panel). Their velocities proved to be $-95 \mathrm{~km} \mathrm{~s}^{-1}$ and $-180 \mathrm{~km} \mathrm{~s}^{-1}$. In summer 1999, the red peak also became considerably weaker. Starting from November 1999, the $\mathrm{H} \alpha$ line was very weak for a long time. In December 1999, the absorption component was very deep. At the beginning of 2000, the line had two relatively equal peaks, after which the blue peak weakened and disappeared by summer. The line remained single-peaked, though asymmetric with a variable shape (eighth line in Fig. 3), until the end of 2002. Then it became stronger and developed an absorption component in the centre. During 2003 and 2004 the line was weak and double-peaked (ninth line in Fig. 3), alternatively double-peaked and single-peaked during 2005. In 2005 it was again somewhat stronger and from August 2006 kept strengthening until the beginning of 2007. The shape of the line was double-peaked with the red peak more intense than the blue 


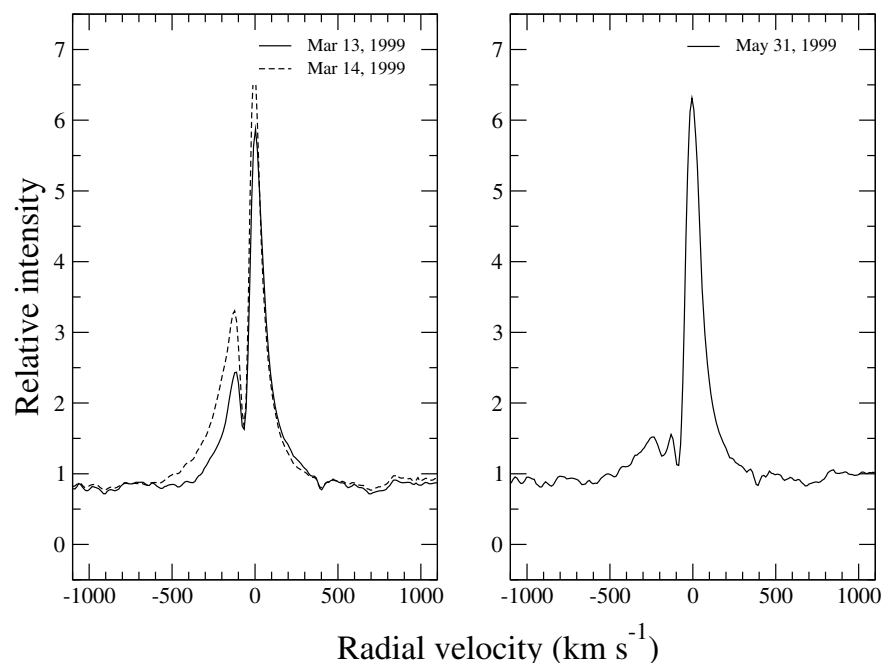

Fig. 4. Left: spectra from two consecutive nights to illustrate the rapid changes of the $\mathrm{H} \alpha$ line in spring 1999. Right: profile with two absorption components.

one (tenth line in Fig. 3). At that time, the visual luminosity of the star reached its minimum at about 10 magnitudes. In spring 2007, the $\mathrm{H} \alpha$ line started to decline and reached the 2004-2006 state by the end of the year.

The resolution of our $\mathrm{H} \beta$ spectra was too low to investigate the shape of the line in any detail. The double-peaked profile in 1998, for instance, observed by Eyres et al. 2002, was not resolved. The measured EWs, however, behaved similarly to those of $\mathrm{H} \alpha$. Once in a while, we also made spectra in the region of $\mathrm{H} \gamma$ and $\mathrm{H} \delta$. These lines are present mainly during active times. No $\mathrm{H} \delta$ emission can be found in our spectra since May 1999, and $\mathrm{H} \gamma$ was at times recogniseable as a very weak red-shifted emission. These appearances tend to be in phase with the maxima of the $\mathrm{H} \alpha$ and $\mathrm{H} \beta$ line strengths.

The measured EWs of $\mathrm{H} \alpha$ and $\mathrm{H} \beta$ are depicted in Figs. 5a and $5 \mathrm{~b}$, respectively. It can be seen that, during quiescence, the EWs of $\mathrm{H} \alpha$ and $\mathrm{H} \beta$ lines change in a wave-like manner. This is even more visible in peak intensities (see Sect. 5.3).

We tried to measure the radial velocities of the $\mathrm{H} \alpha$ line of $\mathrm{CH}$ Cyg by fitting a Gaussian profile to it, though the procedure was often (especially in 1999) complicated by the asymmetric shape of the line. One Gaussian was fitted to the emission line and another to the absorption component (if present). As seen from Fig. 6, both velocities vary with a period of one year. The cause of such periodicity remains a puzzle to us. Velocities of the $\mathrm{H} \beta$ and $\mathrm{H} \gamma$ are plotted in Fig. 7. Generally they accumulate around the system's radial velocity $\left(-59.9 \mathrm{~km} \mathrm{~s}^{-1}\right.$ according to Hinkle et al. 2009), except during a time interval from autumn 1998 to spring 1999 , when they appear to be significantly more positive than usual. This is caused by the very asymmetric profiles of the Balmer lines at that time. The blue-shifted absorption components devoured the blue part of the line and let the red part dominate. The one-year wave does not seem to be present here.

According to the ephemeris by Hinkle et al. (2009), the $\mathrm{CH}$ Cyg system passed the periastron on JD 2451370 (July 1999). Although the proposed eccentricity $(e=0.122)$ is rather small, it could be sufficient to enhance mass transfer from the red giant to the white dwarf, therefore strengthening the Balmer emission lines. This effect is seen especially well in the absolute fluxes (Fig. 9). However, the $\mathrm{H} \alpha$ and $\mathrm{H} \beta$ lines, as well as the (a)

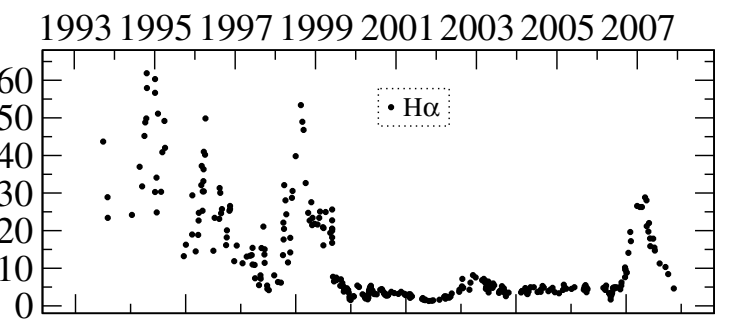

(b)

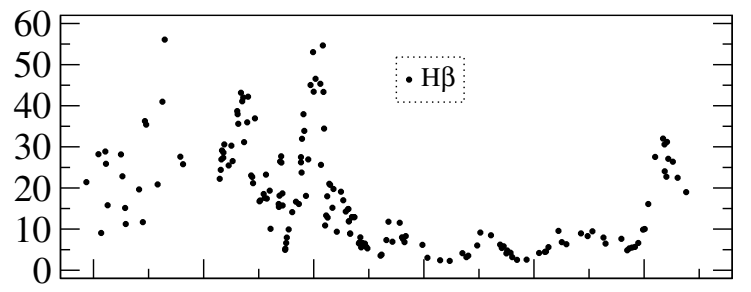

(c)

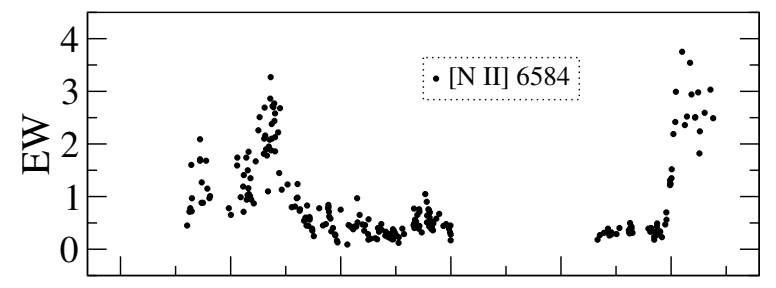

(d)

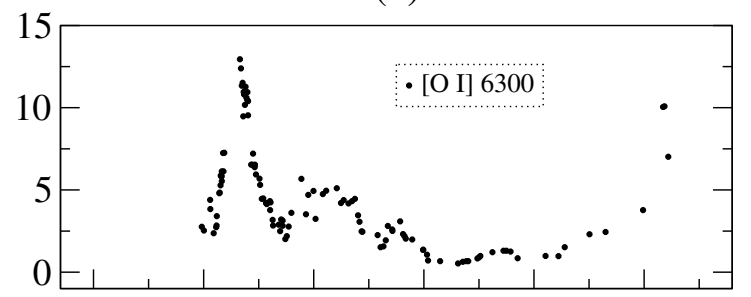

(e)

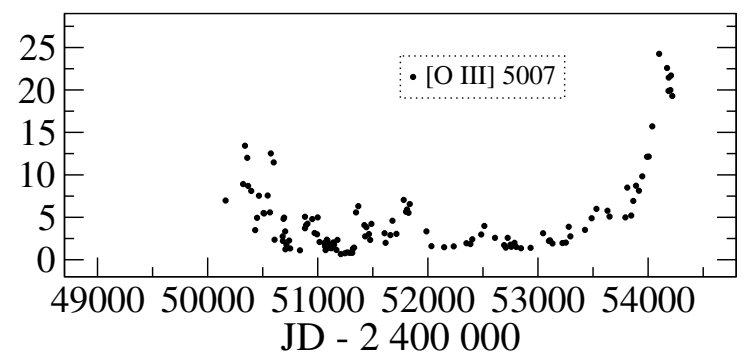

Fig. 5. EWs of some emission lines of $\mathrm{CH}$ Cyg.

[O I] 6300, line reached the maximum intensity a little before the proposed periastron passage, at a phase around 0.95 .

\subsection{Helium lines}

In contrast to most of the symbiotic stars, helium lines are usually weak or absent in the spectrum of $\mathrm{CH} \mathrm{Cyg}$. As found by Leedjärv \& Mikołajewski (2000), the lines of neutral helium $\lambda \lambda 4471,4388$, and 4026 are only visible when the star is brighter than about $10^{\mathrm{m}}$ in the $U$ band. This also applies to our later spectra. In late 1998, when the hydrogen Balmer lines were strong and double-peaked, the above-mentioned He I lines developed P Cygni profiles. In some of our red spectra, He I 6678 was 


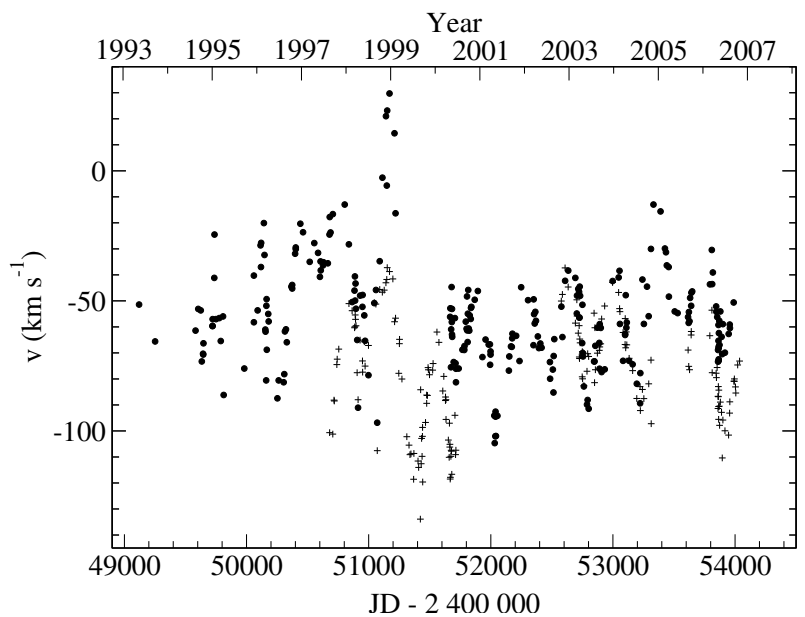

Fig. 6. Velocities of the $\mathrm{H} \alpha$ emission line (filled circles) and its absorption component (crosses).

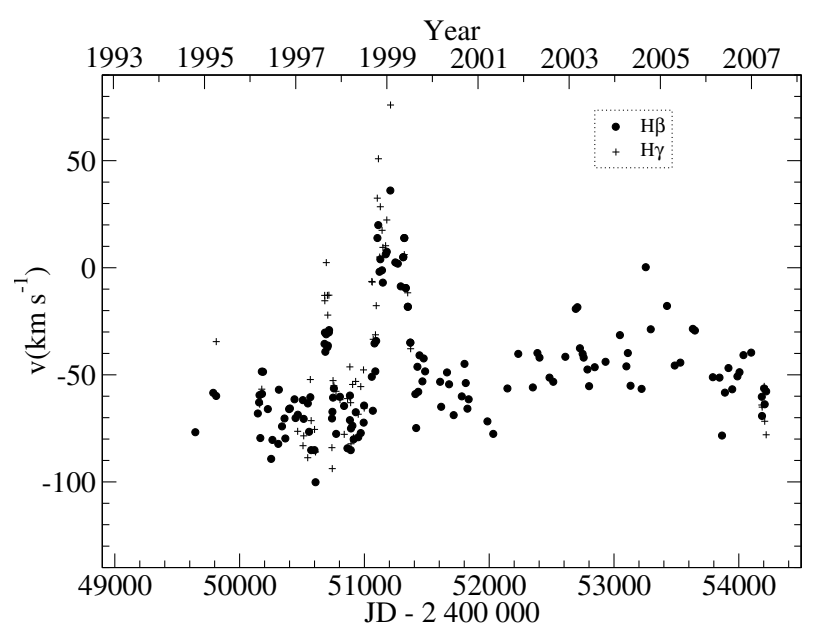

Fig. 7. Velocities of $\mathrm{H} \beta$ and $\mathrm{H} \gamma$.

visible as a faint single-peaked emission. For a short period in 1998-1999 its EW increased to 1 A or more.

There have only been a few incidents when the He II 4686 line, so characteristic of many symbiotics stars, was seen in the spectrum of CH Cyg. In 1984 it was discovered as a very wide emission feature by Leedjärv et al. (1994). In the present spectra, the line was visible for about 100 days in 1998, at the time of brightness maximum $\left(U \sim 8^{\mathrm{m}}\right)$. It appeared for the second time in October 2006, and had at least doubled its strength by the end of the year. In 2007 March 11, when our next spectrum in the blue region was made. The profile of the helium line was much the same (Fig. 8). The line became weaker by summer and was no longer to be found in the spectrum made in October 2007. Interestingly, this appearance of He II 4686 took place during a brightness minimum. $U B V$ photometry shows a steep decrease in light in all bands since the summer of 2006; by the beginning of 2007, the $U$ magnitude had reached $\sim 11.5^{\mathrm{m}}$. We possess no $U B V$ data since that, but the visual AAVSO light curve indicates a new rise from the minimum in the middle of 2007, just about when the helium line disappeared.
He II 4686

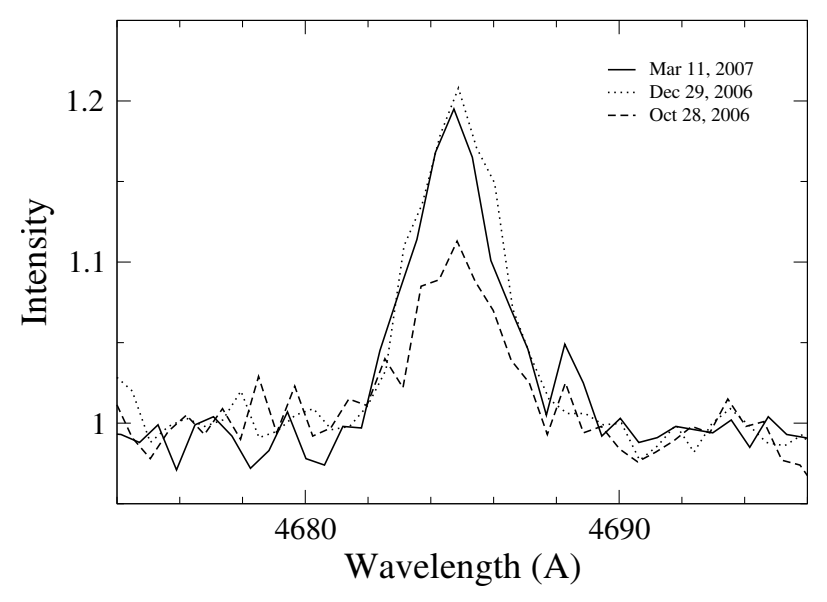

Fig. 8. He II 4686 line.

\section{3. [N II] lines}

As a rule, the lines of [N $\mathrm{NI}] 6548$ and 6584 tend to be absent in S-type symbiotics, but present in D-type systems (Van Winckel et al. 1993). In our spectra of CH Cyg, these lines were visible most of the time.

The behaviour of the EW of [N II] 6584 (the more distinguished of the two) is shown in Fig. 5c. The line was present and quite strong in 1996, but decreased in 1997. It disappeared completely and quite suddenly in spring 2001 and was missing until the end of 2004, when it became barely visible again. During the following year the line was very weak, but detectable. It remained at about the same level until August 2006, when it started to increase steeply. At the end of 2006 and at the beginning of 2007 it was about as strong as in 1996.

The behaviour of [N II] 6548 was similar to 6584. In 1998 the [N II] 6548 line became overwhelmed by the extended blue wing of $\mathrm{H} \alpha$.

The profiles of the $[\mathrm{NII}]$ lines are complicated, especially when the lines are weak, so their velocities are more meaningful when measured at times when the line is stronger. In 1996-1997 the velocities were more negative than in 2006-2007. In the later period the velocities were consistent with those of Balmer lines, about $-60 \mathrm{~km} \mathrm{~s}^{-1}$, which is close to the systemic velocity. During 1996-1997, however, the velocities were about $-100 \mathrm{~km} \mathrm{~s}^{-1}$. H $\alpha$ was stronger in 1996-1997 than it was in 2006-2007, but not so wide as to reach the nitrogen lines, so we do not think it had any effect on their velocities.

\section{4. [OI], [O III], and other forbidden lines}

The forbidden lines [O I] 6300 and 6364 can be found in our lowresolution red spectra. The EWs of these lines seem to follow roughly the course of the Balmer lines (those of [O I] 6300 are depicted in Fig. 5d), except that they have a major maximum during 1996 and the outburst in 1998 is unremarkable.

Our spectra in the $\mathrm{H} \beta$ region include [O III] 4959 and [O III] 5007. The earliest and latest spectra made with HPC-1 and Andor cameras also cover the region of [O III] 4363, and so do the $\mathrm{H} \gamma-\mathrm{H} \delta$ region spectra. This line, however, is not to be found in any of our spectra. [O III] 5007, however, is always present, although it was quite weak from 2001 to 2005. Measured EWs of [O III] 5007 are given in Fig. 5e. [O III] 4959 becomes clearly visible in 2005, remaining much weaker than 5007. As this line 
is located just at the edge of the $\mathrm{TiO} 4955$ absorption band, it is difficult to normalise this spectral region to the local continuum level. For that reason, the measured quantities may be less trustworthy than those of 5007 and we do not present them here, but they do seem to follow the course of those of 5007 line.

On some of our spectra, [S II] 4069 line can be found, as well as [S II] 6731. Its strength appears to behave similarly to [O III] lines. Like [N II] lines, [S II] lines are also usually not found in the spectra of S-type symbiotic stars.

\subsection{Ionised Fe lines}

Our blue spectra made by the Orbis camera span about $4610 \AA$ to $5040 \AA$. This spectral region includes many permitted and forbidden lines of singly ionised iron, like Fe II 4629, [Fe II] 4640, [Fe II] 4729, [Fe II] 4815, [Fe II] 4874, [Fe II] 4890, [Fe II] 4905, Fe II 4924, and Fe II 5018, of which the last two are blended with He I.

In May 1999, most of the listed lines were clearly visible, with the permitted lines stronger than the forbidden ones. Beginning from June, the Fe II lines were mainly gone, except 5018 , but as said, it is not clear how much of a contribution the line gets from He I. [Fe II] lines were not much changed at first and did not begin to fade until autumn.

During the following years, both Fe II and [Fe II] lines were very weak or missing. From the autumn of 2002 we only have one blue spectrum made in November, and on that several Fe lines seem to have strengthened. The intensity of the Fe II lines appeared to reach a local maximum at the beginning of 2003 and that of the [Fe II] lines somewhat later, in April. The Fe II lines did not become as strong as they were in May 1999, but some of the [Fe II] lines even exceeded the intensities they had in 1999.

During 2004, the iron features were rather weak, although the Fe II lines strengthened for a short time in September. During the following year, Fe II lines were basically changeless, [Fe II] seemed to show some maximum in September.

Significant changes took place in the year 2006. The visual brightness of $\mathrm{CH}$ Cyg started to decline in summer, and the intensity of iron lines grew considerably from September on. By the end of the year, the intensities of [Fe II] lines were 3-4 times of their intensities in May 1999. The Fe II lines did not grow to such a degree as to exceed the intensities in May 1999.

In 2007, both Fe II and [Fe II] became step by step weaker.

\subsection{Absolute fluxes}

The appearance of emission lines depends on the level of the continuum: when the continuum is modest, the lines rise more into view; with a strong continuum, they seem weakened. For that reason, conversion of the relative intensity into the absolute fluxes is necessary for obtaining the true picture of line strengths. We gathered the necessary photometric data in $B, V$, and $R$ bands from the literature (see Sect. 3).

As the dates of photometric data usually did not coincide with the dates of our spectra, the values of $B, V$, and $R$ magnitudes were interpolated. When no $R$ values were available, these were computed from $V$ values after finding an approximate relation between $R$ and $V$ magnitudes: $R=0.744 \times V-1.287$. Photometric data were corrected for interstellar extinction using the colour excess $E(B-V)=0.07$ taken from Slovak \& Africano (1978). The computed fluxes are given in Fig. 9 for the same lines as in Fig. 5. (a)

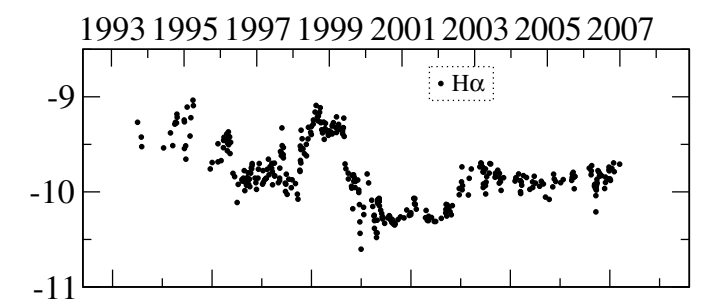

(b)

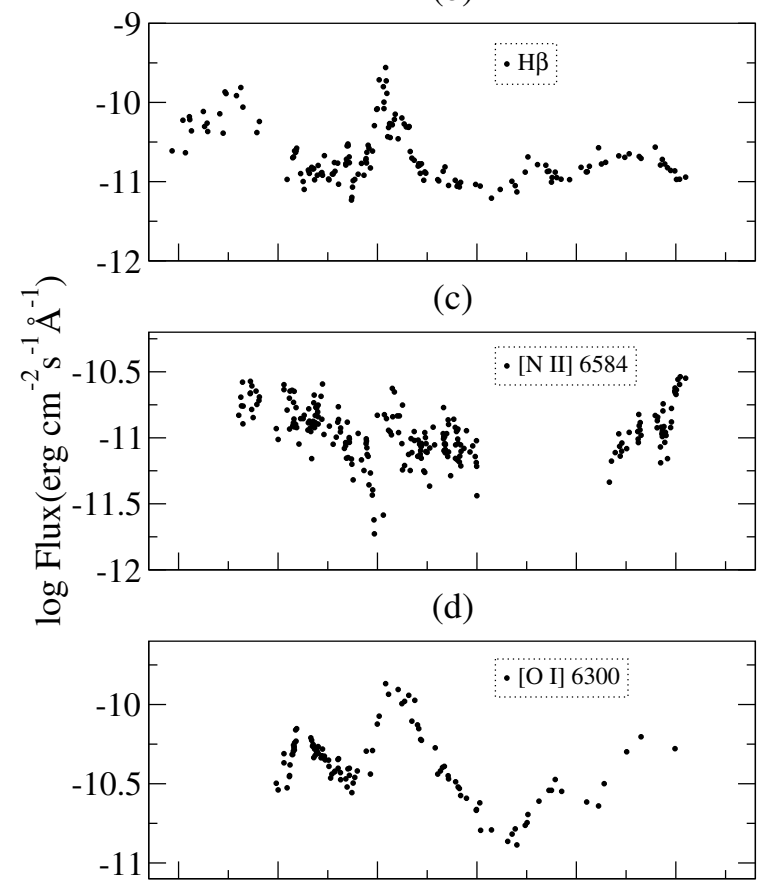

(e)

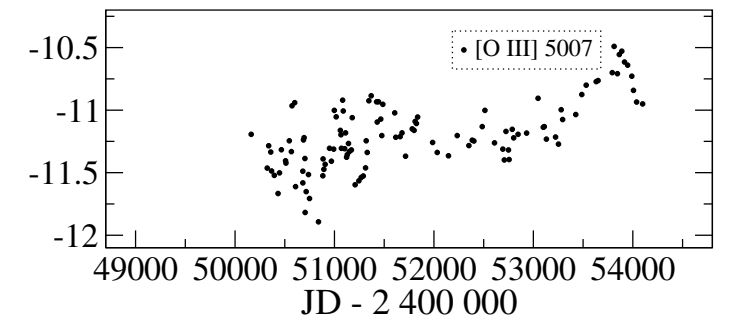

Fig. 9. Fluxes of some emission lines of $\mathrm{CH}$ Cyg.

The outbursts that $\mathrm{CH}$ Cyg had in 1992-1995 and 1998-1999 are reflected in the fluxes and EWs of $\mathrm{H} \alpha$, as well as in the star's light curves. In 1996, when the luminosity has a minimum $\left(V \sim 10^{\mathrm{m}}\right)$, the $\mathrm{EW}$, in contrast, has a maximum (recall the strong single-peaked profiles in 1996). The same happens in 2006, when the luminosity suddenly decreases, EW increases, and the flux, as a result, remains unchanged. This tendency can also be seen in the corresponding figures of $\mathrm{H} \beta$. In the case of [N II] 6584 line, the outbursts do not echo in the EWs, but the minimum-maximum correspondence of continuum and lines in 1996 and 2006 exists here as well. As a consequence, the flux is nearly constant all the time, although the dispersion of the points is large. Our [O I] 6300 data begin at the light maximum of 1996, during which EW of the line becomes high. During the 1998-1999 the EWs only have a small maximum, but the flux increases noticeably. 


\section{Analysis and discussion}

\subsection{Origin of the $\mathrm{H} \alpha$ line}

The interpretation of the $\mathrm{H} \alpha$ line profile depends on how we assume the line is formed. Balmer lines of symbiotic stars are generally thought to originate in that part of the red giant wind that is ionised by the hot component. The double-peaked structure that is often seen in the Balmer lines of symbiotics (van Winckel et al. 1993; Ivison et al. 1994) is probably caused by self-absorption in the neutral hydrogen region around the ionised area. The absorption component tends to be shifted blueward from the line centre. This is caused by the increasing velocity of the wind the absorbing particles in the neutral part of the wind are moving faster towards us than the emitting particles in the underlying ionised region. The depth of the absorption component indicates the density of the wind.

Asymmetric $\mathrm{H} \alpha$ profiles with a blueshifted absorption of different depths prevail during our observations. The line had a similar shape during the 1967-1970 outburst (Faraggiana \& Hack 1971) and 1992-1995 outburst (Skopal et al. 1996a). However, during the longest outburst in 1977-1986 the ratio of the peaks of the $\mathrm{H} \alpha$ line was mostly reversed, as reported by Hack et al. (1986). Robinson et al. (1994) propose that this effect could be caused by the elliptical orbit of the star.

In some symbiotic systems (particularly $\mathrm{T} \mathrm{CrB}$ and CI Cyg, according to Robinson et al. 1994), the double-peaked line profiles could also arise from the accretion disk around the white dwarf, like in the case of cataclysmic variables. It probably cannot be excluded that at times a transient accretion disk may form around the hot component of $\mathrm{CH} \mathrm{Cyg}$ and give rise to the sometimes observed double-peaked profiles.

\subsection{Possible accretion disk in 1998}

We conjecture that, during our observations, the accretion disk could have existed in 1998, when the $\mathrm{H} \alpha$ profile was doublepeaked with almost equal peaks (Fig. 10, upper panel) and had notably wide wings. Fitting the peaks with two Gaussians showed that their positions with regard to the systemic radial velocity varied somewhat, as can be seen from the lower panel of Fig. 10. The depth of the central absorption was about half the height of the peaks at that time. The idea of the line's origin in a disk is supported by the high time resolution photometry by Sokoloski \& Kenyon (2003), who found exceptionally highamplitude flickering in the light curves of CH Cyg in 1998 and also proposed an accretion disk as the source of these. At the same time, the $\mathrm{H} \alpha$ profiles were similar to those in 1982, for which the disk interpretation was successfully used (Leedjärv et al. 1994).

The method of Doppler tomography has been useful for interpreting of the emission lines from accretion disks of cataclysmic variables, but the very long orbital period of $\mathrm{CH}$ Cyg and a short-lived presence of its accretion disk make little use of it here. Nonetheless, we tried to make some estimations of the parameters of the possible disk, based on some assumptions used in Doppler tomography (e.g., Marsh \& Horne 1988; Robinson et al. 1994). The peak-to-peak separation in the line profile in velocity units is assumed to be equal to twice the radial velocity of the material orbiting at the outer edge of the disk. If we know the inclination of the disk and the mass of the accreting star, then from that velocity it is possible to derive the outer radius of the disk (see Leedjärv et al. 1994 for details).
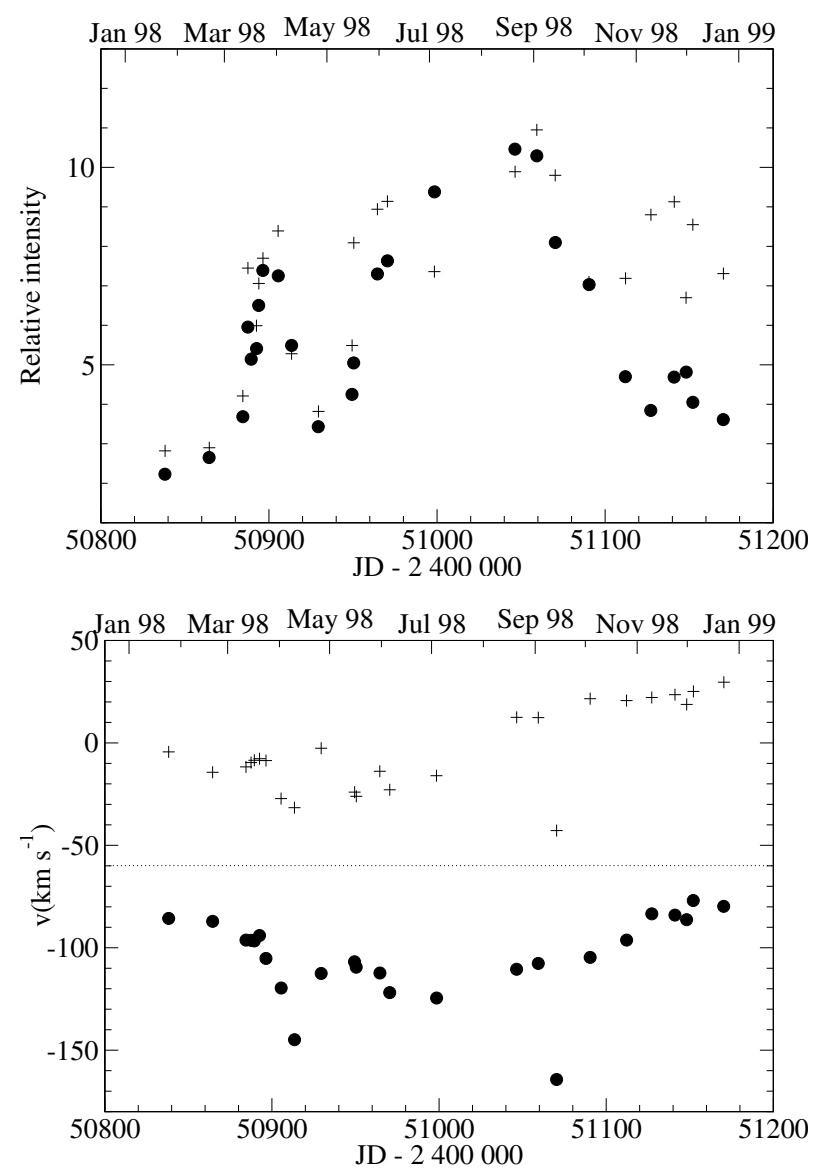

Fig. 10. Top: relative intensities (with respect to the continuum) of the blue and red peaks of $\mathrm{H} \alpha$ in 1998. Bottom: radial velocities of the two peaks. On both graphs, filled circles denote the blue peak and crosses the red peak. The dotted line denotes the systemic velocity.

We assumed the accretion disk to lie in the orbital plane of the system. The system inclination of $\mathrm{CH}$ Cyg is not known, but has to be high enough to allow eclipses. Following the example of Robinson et al. (1994), we adopted the value $i=78^{\circ}$. The mass of the accreting star $M=1 M_{\odot}$ was taken from Mikołajewski \& Mikołajewska (1988). As a result we found that the value of the outer radius falls in the range $(4-9) \times 10^{12} \mathrm{~cm}$, or $50-150 R_{\odot}$. The radial velocities of the peaks in 1984-1987 Leedjärv et al. (1994) were used to estimate the outer radius by the same method and are brought together with our results in Fig. 11. The values of the outer radius seem to flock around $70-75 R_{\odot}$ in both graphs, with the exception of the interval JD 2446 150-350. The results obtained at that time might be an artefact due to the eclipse of the hot component that must have taken place then according to the 5700-d model and to the ephemeris

$\mathrm{JD}($ periastron $)=2445681( \pm 192)+5689.2( \pm 47.0) \times E$

by Hinkle et al. (2009).

\subsection{Variations with the 2.1 year period}

A 2.1-yr period of unclear origin has been repeatedly noticed in the behaviour of CH Cyg. Mikołajewski et al. (1990a) report $a \sim 770$-day periodicity after analysing the long-term optical and near-infrared light curves; $3-5$ deep minima separated by 700-800 days are also found from 1986-1991 multicolour 


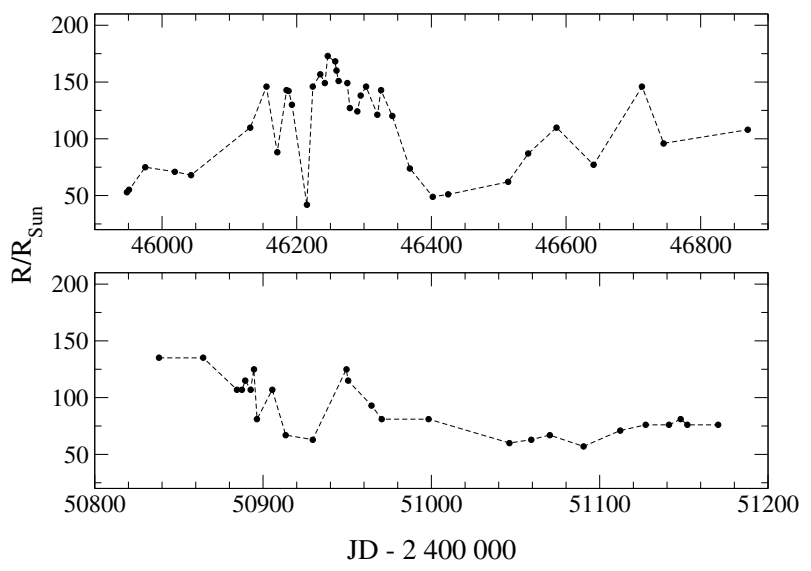

Fig. 11. Radii of the hypothetic accretion disk around the hot component of CH Cyg in 1984-1987 (upper panel) and in 1998 (lower panel).

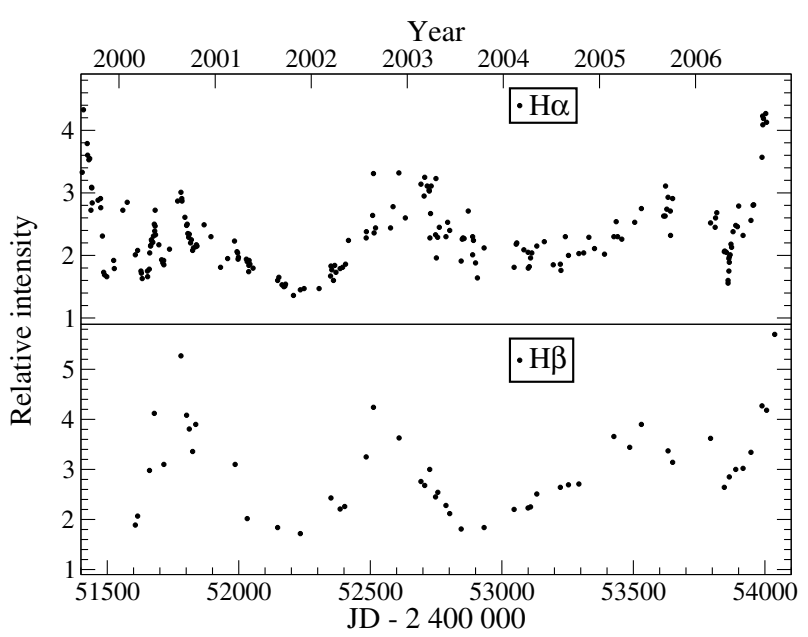

Fig. 12. Intensities of the $\mathrm{H} \alpha$ and $\mathrm{H} \beta$ lines with respect to the continuum during quiescence.

photometry by Mikołajewski et al. (1992). Hinkle et al. (1993) present a 756-day period as the orbital one of the symbiotic system in their triple-star model. Hinkle et al. (2009) confirm the existence of a 2.1-yr period and discuss its two most probable sources: a light companion of the red giant or its nonradial pulsation.

Signs of this period can be found in our spectra. Figure 12 shows the peak intensities of $\mathrm{H} \alpha$ and $\mathrm{H} \beta$ in the time span between the summer of 1999 and the end of 2006, when the lines were weak. Either line shows wavelike behaviour, whereby the minima of the waves correspond to the maxima of the star's light curves, so that the $\mathrm{H} \alpha$ and $\mathrm{H} \beta$ lines are the strongest when the luminosity of the star is the lowest. Visual inspection suggests the period of this variation to be about 2 years. We searched for periodicities from that part of the peak intensity curves and also the corresponding part of EW curves of $\mathrm{H} \alpha$ and $\mathrm{H} \beta$ using phase dispersion minimisation methods. The applied program was ISDA, developed by Dr J. Pelt in Tartu Observatory (see http: //WwW . aai.ee/ pelt/soft.htm\#ISDA). Of the several methods applied, only that of Stellingwerf gave consistent results, which indeed fell near 756 days. The scatter of the points was large, however.

\section{4. $\mathrm{H} \alpha$ lines of $\mathrm{CH}$ Cyg and other symbiotics}

Based on the high-resolution spectra of 59 southern symbiotic and related stars, van Winckel et al. (1993) divided both S- and D-type symbiotics into three subtypes according to the shape and width of their $\mathrm{H} \alpha$ profiles.

Stars of type S-1 have single-peaked asymmetric $\mathrm{H} \alpha$ lines, and those of type $\mathrm{S}-2$ possess a shallow, and those of type S-3 deep absorption components. The $\mathrm{H} \alpha$ lines of D-1 type stars are narrow, single-peaked, and quite symmetric, the lines of type D2 are similar but have a broader base, and type D-3 H $\alpha$ profiles are broad and have an absorption component in the middle.

It is hard to decide into which subtype $\mathrm{CH}$ Cyg fits. Its $\mathrm{H} \alpha$ profile seems to change between S-1 and S-2 subtypes, but the lack or weakness of other lines that characterises $\mathrm{CH}$ Cyg is rather typical of type S-3. On the other hand, we have seen that $\mathrm{CH}$ Cyg sometimes presents rather strong [N II] lines in its spectra. This fact could suggest its belonging to D-type, because these lines are almost never found in the spectra of S-type symbiotics and are very common among D-type systems.

Substantial changes in the shape of the $\mathrm{H} \alpha$ profile have been detected in a few other symbiotics. Van Winckel et al. find that in both S- and D-types of stars, the width of the $\mathrm{H} \alpha$ line and the depth of its absorption component correlate with the strength of the He lines and the visibility of the nebular lines. In the case of $\mathrm{CH}$ Cyg, the strength of other lines changes in phase with the $\mathrm{H} \alpha$ line, but the existence of an absorption component does not seem to play much of a role.

\section{Conclusions}

As a consequence of observing the EWs of the Balmer emission lines of CH Cyg between 1996 to 2007, three maxima were noted: in 1996, in 1998-1999, and in 2006-2007. The first of these was also seen in the EWs of the forbidden lines of [N II], [O I], and, to a lesser extent, [O III]. Comparison with simultaneous photometry revealed, however, that the star's light had a minimum at that time. Therefore, the strengthening of the lines was deceptive, because it was only due to the weakness of the underlying continuum. The second maximum, on the other hand, coincided exactly with the rise and fall of the $U$ light. It only appeared in the EWs (and fluxes) of Balmer lines; no sign of any strengthening was seen in the forbidden lines. The 2006-2007 maximum in its turn was most conspicuous in the EWs of the forbidden lines. Like the 1996 event, it also took place during brightness minimum. The fluxes of Balmer lines show no maximum, those of the forbidden lines, however, are remarkably high. The [O III] lines have been previously noticed as accompanying mass outflows, on account of what we suspect the presence of extended emissions at that time. Regrettably there have so far been no reports of radio mapping since 2000, infrared imaging since 2003, or optical imaging since 1999.

Intensive accretion to the white dwarf was presumably responsible for the 1998 event. The outburst was observable in the Balmer lines, which are supposed to form near the accretor, but undetected in the forbidden lines, arising in a tenuous environment farther away. The strong double-peaked profile of the $\mathrm{H} \alpha$ line, supported by observations of flickering, suggests the presence of an accretion disk around the white dwarf at that time. The outer radius of such a disk was estimated to be about 70-75 $R_{\odot}$. The disk was likely disrupted in 1999 , when the $\mathrm{H} \alpha$ and $\mathrm{H} \beta$ lines rapidly decayed and flickering disappeared (Eyres et al. 2002). The similarity of the $\mathrm{H} \alpha$ profiles in 1982-1983 (Leedjärv et al. 1994) and 1998-1999 seems to confirm the orbital period 
$5689^{d}$ and an elliptical orbit proposed by Hinkle et al. (2009). Both episodes of symmetric double-peaked profiles took place slightly before the periastron passage.

For more than half the period of our observations (in particular between 2000 and 2006), both the luminosity and the strength of the emission lines remained on an approximately constant, low level. The star did not completely lose its symbiotic features, though, as the [O I] and [O III] lines remained visible. The 756-day period, which has previously been seen in the infrared velocities, was found in our Balmer lines' intensities at that time. It is possible that this wave, like the increases in 1996 and 2006-2007 is caused by the changes in the underlying continuum; in this case, however, it is unclear why its amplitude is even slightly larger in $\mathrm{H} \beta$ data.

There are a few more symbiotic stars known whose hot components have a luminosity below or around $100 L_{\odot}$, e.g. MWC 560, R Aqr, EG And, BX Mon. The first two are wellknown jet sources, while both $\mathrm{CH}$ Cyg and $\mathrm{R}$ Aqr also have shown X-ray jets (Kellogg et al. 2007). Shugarov et al. (2007) link V407 Cyg to these objects and propose that a new subclass of symbiotic stars could exist with $\mathrm{CH}$ Cyg, V407 Cyg, and R Aqr as prototypes. A characteristic feature of those stars would be an accretion disk around the white dwarf, which gives rise to flickering on short time scales and occasionally causes the ejection of jets. Most likely those stars have longer orbital periods than do S-type symbiotic stars.

Acknowledgements. The authors wish to thank K. Annuk, T. Eenmäe, A. Hirv, and A. Puss, who did a large part of the observations. The work was partly supported by the Estonian Science Foundation grant No. 6810, and by the target-financed project SF0060030s08 financed by the Ministry of Education and Research of Estonia.

\section{References}

Belczyński, K., Mikołajewska, J., Munari, U., Ivison, R. J., \& Friedjung, M. 2000, A\&AS, 146, 407

Biller, B. A., Close, L. M., Li, A., et al. 2006, ApJ, 647, 464

Brocksopp, C., Sokoloski, J. L., Kaiser, C., et al. 2004, MNRAS, 347, 430

Crocker, M. M., Davis, R. J., Eyres, S. P. S., et al. 2001, MNRAS, 326, 781

Deutsch, A. J. 1964, Ann. Rept. Mt. Wilson and Palomar Obs. 1963, 11

Dobrzycka, D., Kenyon, S. J., \& Milone, A. E. 1996, AJ, 111, 414

Eyres, S. P. S., Bode, M. F., Skopal, A. et al. 2002, MNRAS, 335, 526

Ezuka, H., Ishida, M., \& Makino, F. 1998, ApJ, 499, 388
Faraggiana, R., \& Hack, M. 1971, A\&A, 15, 55

Galloway, D. K., \& Sokoloski, J. L. 2004, ApJ, 613, L61

Hack, M., Rusconi, L., Sedmak, G., et al. 1986, A\&A, 159, 117

Hinkle, K. H., Fekel, F. C., Johnson, D. S., \& Scharlach, W. W. G. 1993, AJ, 105,1074

Hinkle, K. H., Fekel, F. C., \& Joyce, R. 2009, ApJ, 692, 1360

Hric, L., Skopal, A., Urban, Z., et al. 1996, CoSka, 26, 46

Ivison, R. J., Bode, M. F., \& Meaburn, J. 1994, A\&AS, 103, 201

Karovska, M., Carilli, C. L., \& Mattei, J. A. 1998, JAVSO, 26, 97

Karovska, M., Carilli, C., L., Raymond, J. C., \& Mattei, J. A. 2007, ApJ, 661, 1048

Kellogg, E., Anderson, C., Korreck, K., et al. 2007, ApJ, 664, 1079

Leedjärv, L. 2004, BaltA, 13, 109

Leedjärv, L. \& Mikołajewski, M. 2000, ASPC, 204, 345

Leedjärv, L., Mikołajewski, M., \& Tomov, T. 1994, A\&A, 287, 543

Marsh, T. R., \& Horne, K. 1988, MNRAS, 235, 269

Mikołajewski, M., \& Mikołajewska, J. 1988, in The Symbiotic Phenomenon, ed. J. Mikolajewska, M. Friedjung, S. J. Kenyon, \& R. Viotti (Dordrecht: Reidel, IAU Coll., 103, 233

Mikołajewski, M., \& Tomov, Y. 1986, MNRAS, 219, 13P

Mikołajewski, M., Mikołajewka, J., \& Khudyakova, T. N. 1990a, A\&A, 235, 219

Mikołajewski, M., Mikołajewska, J., Tomov, T., Kulesza, B., \& Szczerba, R. 1990b, AcA, 40, 129

Mikołajewski, M., Mikołajewska, J., \& Khudyakova, T. N. 1992, A\&A, 254, 127

Mikołajewski, M., Tomov, T. V., Kolev, D., \& Leedjä, L. 1996, IBVS, 4368

Mukai, K., Ishida, M., Kilbourne, C., et al. 2007, PASJ, 59, 177

Munari, U., Yudin, B. F., Kolotilov, E. A., \& Tomov, T. V. 1996, A\&A, 311, 484

Munari, U., Renzini, A., \& Bernacca, B. L. 1997, Hipparcos-Venice '97 (ESA SP-402), 413

Munari, U., \& Zwitter, T. 2002, A\&A, 383, 188

Robinson, K., Bode, M. F., Skopal, A., Ivison, R. J., \& Meaburn, J. 1994, MNRAS, 269, 1

Shugarov, S. Yu., Tatarnikova, A. A., Kolotilov, E. A., Shenavrin, V. I., \& Yudin, B. F. 2007, BaltA, 16, 23

Skopal, A. 1995, IBVS, 4157

Skopal, A. 1997, IBVS, 4495

Skopal, A. 1998, CoSka, 28, 87

Skopal, A., Hric, L., Chochol, D., et al. 1995, CoSka, 25, 53

Skopal, A., Bode, M. F., Bryce, M., et al. 1996a, MNRAS, 282, 327

Skopal, A., Bode, M.F., Lloyd, H. M., \& Tamura, S. 1996b, A\&A, 308, L9

Skopal, A., Pribulla, T., Wolf, M., et al. 2000, CoSka, 30, 29

Skopal, A., Vaňko, M., Pribulla, T., et al. 2002, CoSka, 32, 62

Skopal, A., Pribulla, T., Vaňko, M., et al. 2004, CoSka, 34, 45

Skopal, A., Vaňko, M., Pribulla, T., et al. 2007, AN, 328, 909

Slovak, M. H., \& Africano, J. 1978, MNRAS, 185, 591

Sokoloski, J. L., \& Kenyon, S. J. 2003, ApJ, 584, 1027

Solf, J. 1987, A\&A, 180, 207

Taranova, O., \& Shenavrin, V. 2006, PZP, 6, 28

Taylor, A. R., Seaquist, E. R., \& Mattei, J. A. 1986, Nature, 319, 38

van Winckel, H., Duerbeck, H. W., \& Schwarz, H. E. 1993, A\&AS, 102, 401

Wheatley, P. J., \& Kallman, T. R. 2006, MNRAS, 372, 1602

Yamashita, Y., \& Maehara, H. 1979, PASJ, 31, 307 